\title{
The effect of environmental factors on the mayfly communities of headwater streams in the Pieniny Mountains (West Carpathians)
}

\author{
Małgorzata KŁONOWsKa-OlejniK ${ }^{1} \&$ Tomasz SkAlSkI $^{2}$ \\ ${ }^{1}$ Friedleina 33/19, 30-009 Kraków, Poland; e-mail: uxklonow@cyf-kr.edu.pl \\ ${ }^{2}$ Institute of Zoology, Jagiellonian University, Gronostajowa 9, 30-387 Kraków, Poland; e-mail: tomasz.skalski@uj.edu.pl
}

\begin{abstract}
A study of the species composition of mayfly communities in connection with environmental parameters was made in headwater streams of the Pieniny Mts. The rhithral zone is inhabited maximally by 19 mayfly species. In most of the streams studied the mayfly communities were found to be similar, however the vertical zonation which reflected human impact was visible (NMDS analysis). The main factors responsible for mayfly communities at all the sites studied were stream regulation and organic pollution, followed by type of bottom substrate (pebble and gravel), riparian vegetation (shrubs), $\mathrm{pH}$ and water temperature. At undisturbed sites the most important factors were $\mathrm{pH}$, substrate type, distance from the source, current velocity and riparian vegetation (CCA analysis). Analysis of mayfly communities and environmental characteristics in different seasons showed that occurrence of mayfly species varied substantially depending on the season. Only in early spring and autumn do mayfly communities occur which are dependent on many environmental factors, the most significant of which are substrate type, phosphate, distance from source and altitude (CCA analysis).
\end{abstract}

Key words: mayfly communities; headwater stream; environmental conditions; seasonal variation

\section{Introduction}

Mayflies (Ephemeroptera), which are one of the main groups of freshwater macroinvertebrates, are present in almost all types of running waters. Due to longitudinal zonation of streams, macroinvertebrate communities, their structure and functional feeding group change with the progression from springs to streams and rivers (Vannote et al. 1980; Minshall et al. 1985). The longitudinal distribution of macroinvertebrates is determined by many of environmental parameters on the longitudinal gradient. The main abiotic factors are altitude (Finn \& Poff 2005), slope (Breitenmoser-Würsten \& Sartori 1995), stream size (Heino et al. 2005), development of riparian zone and its vegetation (Roth et al. 1996; Townsend et al. 2003), current velocity (Burian 1997), substrate type and size (Hawkins et al. 1982), water chemistry and nutrient concentration (Krno et al. 2007) and water temperature (Ward 1992).

Apart from natural factors, human impact also plays a significant role. Many streams are threatened by organic pollution (Zamora-Muńoz et al. 1993). Stream regulation causes numerous changes, mainly in flow regulation (Bunn \& Arthington 2002) and channel and bank degradation (Lenzi \& Comiti 2003). These factors are responsible for negative influence on species diversity and domination of generalist species in the communities.

The river continuum concept and longitudinal distribution of freshwater macroinvertebrate communities are not always and only locally confirmed in freshwater ecosystems (Lorenz et al. 2004), particularly in highmountain streams (Krno et al. 2006). Local environmental conditions (local and regional characteristics) seem to be very important for macroinvertebrate assemblages on a regional scale, in particular catchment areas (Death \& Joy 2004; Krno et al. 2007). Geographical location also influences invertebrate communities (Townsend et al. 2003), particularly in terms of geology and geomorphology (Johnson \& Gage 1997; Hieber et al. 2005).

All of the streams studied belong to Pieniny National Park, a protected area. Streams in the Pieniny Mts are largely natural, but some have been subject to human impact. Mayfly communities in this region had not previously been researched (Kłonowska-Olejnik 2000). Due to the distinctive abiotic conditions in this area (short, shaded streams with low water temperature), distinctive mayfly assemblages may also be expected.

Investigation of the natural or semi-natural macroinvertebrate communities of the streams in this area is essential for characteristic these communities and their longitudinal gradient. Furthermore, it has practical applications for understanding reference conditions for biomonitoring. Determination of the environmental parameters influencing mayfly community composition makes it possible to predict the composition of, and changes in, communities of particular streams. The distinctive local conditions determining mayfly communi- 


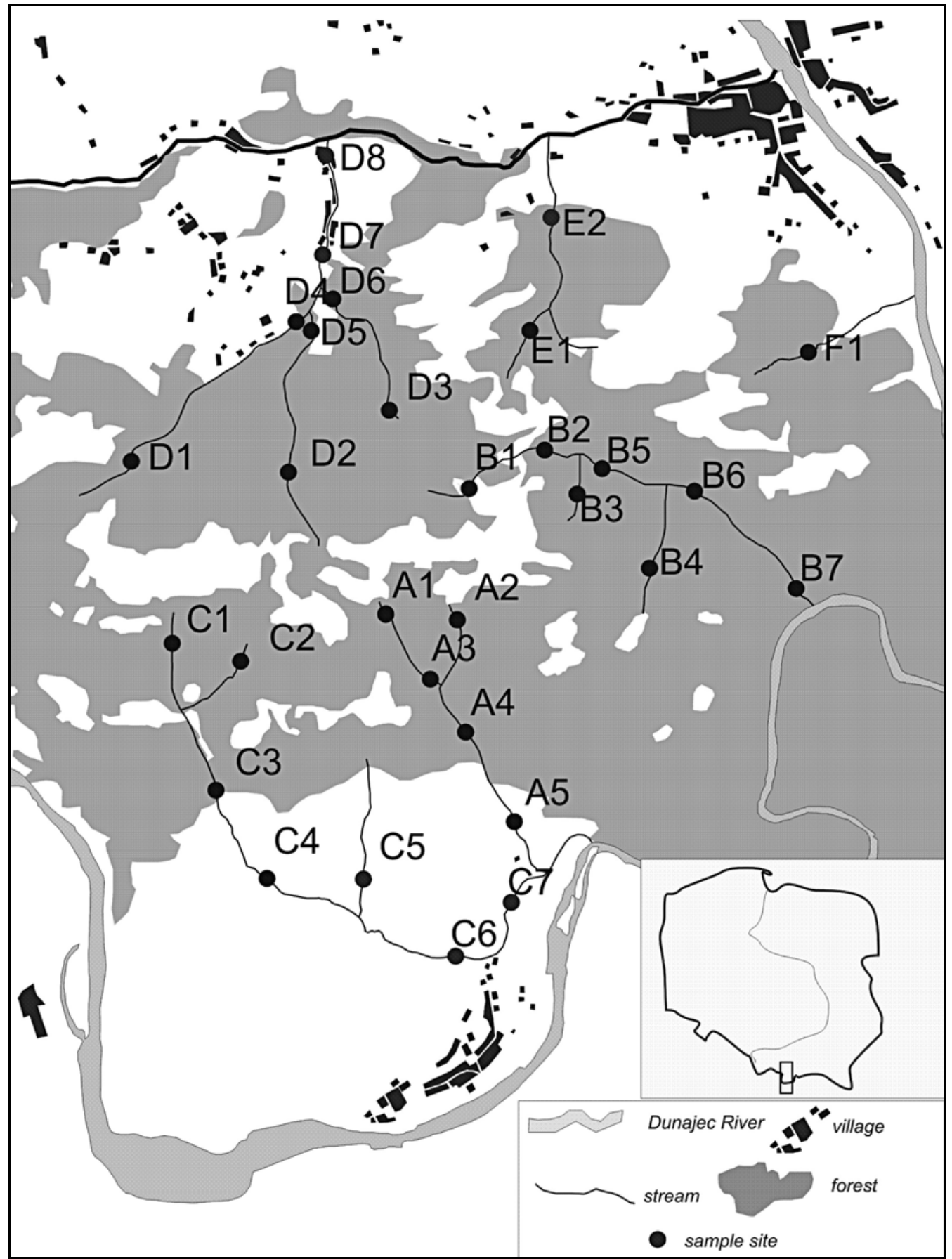

Fig. 1. Map of the Pieniny Mts showing sampling sites: A - Sobczyński stream sites; B - Pieniński stream sites; C - Macelowy stream sites; D - Biały stream sites; E - Łonny stream sites; F - Ociemny stream sites.

ties are very often overlooked, and their lack of diversity is mistakenly interpreted as disturbances caused by anthropogenic factors. Understanding mayfly community structure is therefore important for bioassessment and conservation.

The aims of the study were as follows: (1) To describe the mayfly communities of headwater streams in the Pieniny Mts and to examine the variation between them; (2) To identify the abiotic parameters that best define the composition of mayfly communities; (3) To determine in which way mayfly communities are subject to seasonal changes.

\section{Material and methods}

\section{Study area}

The study area was in the Pieniny Mts in southern Poland, which are composed mainly of soft limestone, chalk marl and Jurassic limestone. Thirty sampling sites were selected along 6 streams - all of the major streams of Pieniny National Park, a protected area in the Pieniny Mts. All sites were in headwater streams, first to fourth order, crenal and rithral, at elevations between $750 \mathrm{~m}$ a.s.l. and $440 \mathrm{~m}$ a.s.l. Their distribution is shown in Fig. 1. Most of the sampling sites were natural or semi-natural, with minimal human impact. Some of the sites were in streams with anthropogenic disturbance: organic pollution, stream regu- 
Table 1. Environmental characteristics of the study sites.

\begin{tabular}{|c|c|c|c|c|c|c|c|c|c|c|c|c|c|c|c|c|c|c|c|}
\hline Site & Alt & Dis & Slo & Wid & Dep & Vel & B & $\mathrm{C}$ & $\mathrm{PG}$ & SA & $\mathrm{M}$ & Org & $\mathrm{Md}$ & $\mathrm{O}$ & $\mathrm{R}$ & $\mathrm{D}$ & $\mathrm{F}$ & $\mathrm{S}$ & $\mathrm{G}$ \\
\hline A_1 & 750 & 0.09 & 0.22 & 0.75 & 0.06 & 1 & 5 & 30 & 65 & 0 & 0 & 30 & 1 & 0 & 0 & 1 & 90 & 0 & 10 \\
\hline A_3 & 600 & 0.5 & 0.28 & 0.75 & 0.1 & 2 & 5 & 20 & 75 & 0 & 0 & 10 & 1 & 0 & 0 & 1 & 100 & 0 & 0 \\
\hline A_2 & 675 & 0.08 & 0.27 & 0.4 & 0.1 & 1 & 0 & 40 & 60 & 0 & 0 & 25 & 0 & 0 & 0 & 0 & 60 & 0 & 40 \\
\hline A_4 & 550 & 0.83 & 0.27 & 1.75 & 0.2 & 3 & 30 & 40 & 40 & 0 & 0 & 20 & 0 & 0 & 0 & 0 & 0 & 70 & 30 \\
\hline A_5 & 470 & 1.38 & 0.22 & 1.5 & 0.15 & 3 & 10 & 50 & 40 & 0 & 0 & 30 & 0 & 0 & 0 & 0 & 0 & 80 & 20 \\
\hline B_1 & 710 & 0.23 & 0.13 & 1.25 & 0.08 & 1 & 0 & 15 & 70 & 10 & 5 & 60 & 0 & 0 & 0 & 0 & 100 & 0 & 0 \\
\hline B_2 & 660 & 0.7 & 0.12 & 1 & 0.12 & 2 & 5 & 20 & 70 & 5 & 0 & 40 & 0 & 0 & 0 & 0 & 100 & 0 & 0 \\
\hline B_5 & 610 & 0.95 & 0.14 & 2.25 & 0.15 & 3 & 5 & 15 & 65 & 5 & 10 & 50 & 0 & 0 & 0 & 0 & 100 & 0 & 0 \\
\hline B_6 & 535 & 1.45 & 0.14 & 2 & 0.18 & 3 & 10 & 30 & 50 & 5 & 5 & 20 & 0 & 0 & 0 & 0 & 100 & 0 & 0 \\
\hline B_7 & 440 & 2.23 & 0.13 & 2 & 0.2 & 4 & 10 & 10 & 60 & 10 & 10 & 15 & 0 & 0 & 0 & 0 & 100 & 0 & 0 \\
\hline B_3 & 640 & 0.15 & 0.27 & 1.5 & 0.15 & 1 & 5 & 5 & 80 & 5 & 5 & 40 & 0 & 0 & 0 & 0 & 100 & 0 & 0 \\
\hline B_4 & 620 & 0.39 & 0.29 & 1.25 & 0.1 & 1 & 5 & 5 & 80 & 5 & 5 & 30 & 0 & 0 & 0 & 0 & 100 & 0 & 0 \\
\hline C_1 & 680 & 0.18 & 0.29 & 1.25 & 0.1 & 1 & 5 & 15 & 80 & 0 & 0 & 20 & 1 & 0 & 0 & 1 & 100 & 0 & 0 \\
\hline C_2 & 660 & 0.28 & 0.29 & 1 & 0.1 & 1 & 10 & 30 & 60 & 0 & 0 & 30 & 0 & 0 & 0 & 1 & 100 & 0 & 0 \\
\hline C_3 & 580 & 0.95 & 0.16 & 1 & 0.15 & 2 & 5 & 20 & 70 & 5 & 0 & 10 & 0 & 0 & 0 & 0 & 0 & 100 & 0 \\
\hline C_4 & 520 & 1.54 & 0.14 & 2.25 & 0.18 & 3 & 0 & 5 & 85 & 10 & 0 & 5 & 1 & 1 & 0 & 0 & 0 & 80 & 20 \\
\hline C_6 & 465 & 2.63 & 0.1 & 2 & 0.25 & 3 & 0 & 5 & 85 & 5 & 5 & 5 & 1 & 1 & 0 & 0 & 0 & 60 & 40 \\
\hline C_7 & 450 & 3.03 & 0.09 & 2 & 0.2 & 3 & 0 & 0 & 90 & 5 & 5 & 5 & 2 & 1 & 1 & 0 & 0 & 10 & 90 \\
\hline C_5 & 500 & 0.65 & 0.25 & 1.25 & 0.1 & 2 & 5 & 20 & 70 & 0 & 5 & 5 & 0 & 0 & 0 & 0 & 0 & 20 & 80 \\
\hline D_1 & 660 & 0.33 & 0.15 & 0.75 & 0.08 & 1 & 30 & 50 & 20 & 0 & 0 & 30 & 1 & 0 & 0 & 1 & 100 & 0 & 0 \\
\hline D_4 & 620 & 1.48 & 0.06 & 2 & 0.15 & 3 & 10 & 20 & 70 & 0 & 0 & 20 & 2 & 1 & 1 & 0 & 60 & 20 & 20 \\
\hline D_2 & 660 & 0.35 & 0.2 & 0.75 & 0.08 & 2 & 30 & 60 & 10 & 0 & 0 & 40 & 0 & 0 & 0 & 0 & 100 & 0 & 0 \\
\hline D_5 & 520 & 1.15 & 0.18 & 1.5 & 0.12 & 3 & 10 & 10 & 70 & 5 & 5 & 20 & 0 & 0 & 0 & 0 & 60 & 20 & 20 \\
\hline D_7 & 500 & 1.88 & 0.11 & 1.75 & 0.2 & 4 & 5 & 10 & 80 & 5 & 0 & 20 & 3 & 1 & 1 & 1 & 40 & 50 & 10 \\
\hline D_8 & 465 & 2.45 & 0.1 & 3 & 0.15 & 5 & 0 & 0 & 0 & 0 & 0 & 0 & 3 & 1 & 1 & 1 & 0 & 20 & 80 \\
\hline D_3 & 660 & 0.09 & 0.22 & 0.75 & 0.08 & 1 & 5 & 10 & 85 & 0 & 0 & 30 & 0 & 0 & 0 & 0 & 100 & 0 & 0 \\
\hline D_6 & 520 & 0.79 & 0.2 & 1.75 & 0.15 & 3 & 5 & 10 & 75 & 5 & 5 & 20 & 1 & 0 & 0 & 1 & 100 & 0 & 0 \\
\hline E_1 & 560 & 0.28 & 0.22 & 1.75 & 0.8 & 1 & 10 & 10 & 80 & 0 & 0 & 30 & 0 & 0 & 0 & 0 & 100 & 0 & 0 \\
\hline E_2 & 465 & 0.95 & 0.16 & 2 & 0.15 & 3 & 5 & 10 & 80 & 5 & 0 & 20 & 0 & 0 & 0 & 0 & 80 & 20 & 0 \\
\hline F_1 & 500 & 0.33 & 0.22 & 0.75 & 0.8 & 2 & 0 & 5 & 90 & 5 & 0 & 20 & 0 & 0 & 0 & 0 & 100 & 0 & 0 \\
\hline
\end{tabular}

Explanations: A - Sobczyński stream sites; B - Pieniński stream sites; C - Macelowy stream sites; D - Biały stream sites; E - Łonny stream sites; F - Ociemny stream sites; Alt - Altitude (m a.s.l.); Dis - distance from source $(\mathrm{m})$; Slo - slope $\left(\mathrm{m} \mathrm{m}^{-1}\right)$; Wid - stream mean width (m); Dep - mean water depth (m); Vel - current velocity; B - boulder (\%); C - cobble (\%); PG - pebble and gravel (\%); $\mathrm{SA}$ - sand (\%); M - mud (\%); Org - organic matter (\%); Md - man disturbance; O - organic pollution; R - stream regulation; D channel degradation by forest land use; $\mathrm{F}$ - forest (\% of riparian vegetation cover); $\mathrm{S}$ - shrubs (\% of riparian vegetation cover); $\mathrm{G}-$ grasses (\% of riparian vegetation cover). For more details see text.

lation and channel degradation resulting from forest land use.

\section{Sampling}

Mayfly larvae were sampled using a Surber sampler (frame size: $0.25 \mathrm{~m} \times 0.25 \mathrm{~m}$, mesh size $300 \mu \mathrm{m})$. Benthic samples were collected using the kick-method. Each sample was taken of all microhabitat and substrate types within a 10 $\mathrm{m}$ reach of stream at each site. The sites were sampled 4 times (early spring, late spring, summer and autumn), in 2008 and 2009. Five samples of benthic macroinvertebrates were taken each time at each site. Samples were preserved in the field with $4 \%$ formaldehyde and taken to a laboratory, where the material was sorted and preserved in $75 \%$ ethyl alcohol. All mayfly larvae were counted and identified to the lowest possible taxonomic level (species or genus). Most of the material was identified to species, but early instar larvae were identified to the genus level. Mayfly larvae abundance was calculated as individuals per $\mathrm{m}^{2}$. Each sampling site was characterized using 19 environmental variables (see Table 1). Some environmental characteristics were determined from topographic maps (1:10 000) (altitude, distance from source, slope). Stream width and mean water depth were calculated based on three measurements at each site. Water velocity was determined by moving a bobber over a distance of $10 \mathrm{~m}$ (3 times) (Gordon et al. 1994) and ranged from 1 to 5 (current velocity 1 : less than $0.05 \mathrm{~m} \mathrm{~s}^{-1} ; 2$ : $0.05-0.25$ $\mathrm{m} \mathrm{s}^{-1} ; 3: 0.25-0.5 \mathrm{~m} \mathrm{~s}^{-1} ; 4: 0.5-1.0 \mathrm{~m} \mathrm{~s}^{-1} ; 5$ : more than $\left.1.0 \mathrm{~m} \mathrm{~s}^{-1}\right)$. Substrate composition was determined as the percentage of each fraction at each sampling site. Substratum particle sizes were determined using Wolman's granulometry standard method (Wolman 1954). Organic detritus was estimated as a percentage of the bottom coverage at each sampling site. Riparian vegetation on the bank was estimated visually as percentages of trees $(2-10 \mathrm{~m})$, shrubs $(0.5-2 \mathrm{~m})$ and herbs $(0.1-0.5 \mathrm{~m})$ in the immediate vicinity of the stream. Channelization and channel degradation resulting from forest land use were visually estimated at each site. A detailed description of the environmental characteristics of the sampling sites is given in Table 1. On each sampling date we also recorded physical and chemical water characteristics. Some water parameters (temperature, conductivity, $\mathrm{pH}$, dissolved oxygen) were measured in the field using a Hanna HI991300 pH/EC/TDS/Temperature meter and a Hanna H9143 Dissolved Oxygen meter. In addition, water samples were taken to the laboratory and analysed for total hardness, ammonium, nitrite, nitrate and phosphate in a Hanna HI83200 Multiparameter Photometer. Physical and chemical data are given in Table 2 .

\section{Statistical analyses}

Indirect ordination of the mayfly communities found at the 30 sites was performed using non-metric multidimensional scaling (NMDS). NMDS was calculated in WinKyst 1.0 (Šmilauer 2002) on a Bray-Curtis similarity matrix, based on an initial configuration generated by principal coordinate analysis. The plot was subsequently orientated us- 
Table 2. Chemical and physical characteristics of the study sites.

\begin{tabular}{|c|c|c|c|c|c|c|c|c|c|c|c|c|c|c|c|c|c|c|}
\hline \multirow{2}{*}{ Site } & \multicolumn{2}{|c|}{$\mathrm{T}$} & \multicolumn{2}{|c|}{$\mathrm{pH}$} & \multicolumn{2}{|c|}{ Cond } & \multicolumn{2}{|c|}{ Har } & \multicolumn{2}{|c|}{ Oxy } & \multicolumn{2}{|c|}{$\mathrm{NH}_{4}-\mathrm{N}$} & \multicolumn{2}{|c|}{$\mathrm{NO}_{2}-\mathrm{N}$} & \multicolumn{2}{|c|}{$\mathrm{NO}_{3}-\mathrm{N}$} & \multicolumn{2}{|c|}{ Phos } \\
\hline & Mean & $\mathrm{SD}$ & Mean & $\mathrm{SD}$ & Mean & $\mathrm{SD}$ & Mean & $\mathrm{SD}$ & Mean & $\mathrm{SD}$ & Mean & $\mathrm{SD}$ & Mean & $\mathrm{SD}$ & Mean & $\mathrm{SD}$ & Mean & SD \\
\hline A_1 & 10.2 & 4.4 & 7.1 & 0.7 & 241.0 & 14.2 & 182.5 & 23.6 & 8.30 & 0.60 & 0.53 & 0.78 & 0.07 & 0.03 & 5.90 & 0.36 & 0.00 & 0.00 \\
\hline A_2 & 10.4 & 3.6 & 6.9 & 1.1 & 289.3 & 8.4 & 173.8 & 7.5 & 6.80 & 1.94 & 0.28 & 0.17 & 0.04 & 0.03 & 4.00 & 1.44 & 0.03 & 0.05 \\
\hline A_3 & 12.0 & 4.8 & 7.1 & 0.5 & 289.5 & 10.7 & 242.5 & 42.7 & 7.40 & 0.85 & 0.64 & 0.25 & 0.09 & 0.04 & 4.38 & 0.25 & 0.00 & 0.00 \\
\hline A_4 & 11.3 & 5.2 & 6.7 & 0.9 & 279.5 & 10.0 & 205.0 & 25.2 & 7.48 & 1.12 & 0.89 & 1.41 & 0.07 & 0.04 & 3.35 & 1.14 & 0.00 & 0.00 \\
\hline A_5 & 11.1 & 4.2 & 6.8 & 0.7 & 289.0 & 10.4 & 200.0 & 28.3 & 7.88 & 0.32 & 0.09 & 0.05 & 0.03 & 0.02 & 4.83 & 1.79 & 0.00 & 0.00 \\
\hline B_1 & 7.7 & 2.5 & 6.6 & 0.1 & 321.5 & 4.7 & 293.8 & 7.5 & 8.45 & 2.94 & 0.82 & 1.45 & 0.06 & 0.05 & 3.40 & 0.49 & 0.00 & 0.00 \\
\hline B_2 & 8.2 & 3.3 & 6.6 & 0.5 & 314.5 & 2.5 & 311.3 & 53.6 & 9.35 & 1.78 & 0.81 & 1.46 & 0.07 & 0.03 & 3.13 & 0.25 & 0.00 & 0.00 \\
\hline B_3 & 7.5 & 3.3 & 6.5 & 0.6 & 276.3 & 4.7 & 258.8 & 42.1 & 9.30 & 2.07 & 0.81 & 1.46 & 0.06 & 0.05 & 1.65 & 0.83 & 0.00 & 0.00 \\
\hline B_4 & 7.4 & 4.2 & 6.6 & 0.6 & 270.3 & 1.0 & 257.5 & 53.2 & 9.20 & 1.97 & 0.79 & 1.48 & 0.06 & 0.05 & 14.00 & 1.92 & 0.00 & 0.00 \\
\hline B_5 & 7.8 & 3.7 & 6.7 & 0.4 & 299.5 & 3.9 & 260.0 & 14.1 & 9.15 & 1.92 & 0.35 & 0.44 & 0.08 & 0.04 & 4.93 & 1.02 & 0.00 & 0.00 \\
\hline B_6 & 8.4 & 4.0 & 6.5 & 1.0 & 273.0 & 3.6 & 242.5 & 5.0 & 9.05 & 2.03 & 0.13 & 0.10 & 0.08 & 0.05 & 7.10 & 1.02 & 0.00 & 0.00 \\
\hline B_7 & 8.9 & 4.1 & 6.3 & 0.7 & 273.8 & 5.2 & 225.0 & 19.1 & 9.18 & 1.92 & 0.83 & 1.45 & 0.28 & 0.42 & 6.43 & 1.90 & 0.00 & 0.00 \\
\hline C_1 & 7.9 & 3.7 & 7.4 & 1.0 & 306.8 & 3.3 & 282.5 & 15.0 & 8.75 & 1.23 & 0.86 & 1.43 & 0.06 & 0.05 & 5.03 & 0.89 & 0.00 & 0.00 \\
\hline C_2 & 7.6 & 3.7 & 6.5 & 0.5 & 292.0 & 20.9 & 215.0 & 19.1 & 8.18 & 0.40 & 0.35 & 0.30 & 0.06 & 0.02 & 4.13 & 0.65 & 0.00 & 0.00 \\
\hline C_3 & 9.8 & 4.2 & 7.3 & 1.0 & 300.3 & 9.4 & 227.5 & 18.9 & 7.78 & 0.74 & 0.82 & 1.45 & 0.08 & 0.04 & 4.55 & 0.68 & 0.00 & 0.00 \\
\hline C_4 & 9.2 & 3.8 & 7.1 & 0.7 & 317.5 & 17.4 & 223.8 & 29.3 & 6.90 & 0.90 & 0.69 & 1.21 & 0.09 & 0.03 & 3.93 & 0.87 & 0.08 & 0.05 \\
\hline C_5 & 9.5 & 4.0 & 7.3 & 0.7 & 319.8 & 3.9 & 210.0 & 25.8 & 8.25 & 1.20 & 0.91 & 1.40 & 0.08 & 0.04 & 2.53 & 0.21 & 0.08 & 0.05 \\
\hline C_6 & 10.3 & 5.0 & 7.1 & 0.8 & 321.5 & 9.4 & 247.5 & 51.2 & 6.98 & 1.24 & 0.54 & 0.71 & 0.19 & 0.21 & 3.93 & 0.19 & 0.10 & 0.07 \\
\hline C_7 & 10.9 & 4.7 & 6.7 & 0.4 & 309.0 & 5.9 & 257.5 & 15.0 & 7.08 & 1.27 & 0.38 & 0.37 & 0.06 & 0.03 & 4.65 & 1.37 & 0.00 & 0.00 \\
\hline D_1 & 7.9 & 4.3 & 7.0 & 0.8 & 274.5 & 2.6 & 238.8 & 41.3 & 8.63 & 2.38 & 0.07 & 0.02 & 0.06 & 0.05 & 5.10 & 0.90 & 0.00 & 0.00 \\
\hline D_2 & 7.7 & 4.6 & 6.7 & 0.5 & 283.8 & 6.7 & 192.5 & 15.0 & 8.90 & 2.16 & 0.05 & 0.00 & 0.06 & 0.06 & 7.18 & 1.53 & 0.00 & 0.00 \\
\hline D_3 & 6.9 & 3.6 & 6.8 & 0.4 & 296.0 & 3.5 & 280.0 & 8.2 & 8.40 & 2.47 & 0.18 & 0.05 & 0.09 & 0.03 & 11.25 & 2.32 & 0.00 & 0.00 \\
\hline D_4 & 8.4 & 4.0 & 6.8 & 0.7 & 310.0 & 15.2 & 275.0 & 61.4 & 7.35 & 3.49 & 0.81 & 1.46 & 0.11 & 0.02 & 6.05 & 0.87 & 0.11 & 0.02 \\
\hline D_5 & 8.5 & 4.5 & 6.8 & 0.7 & 293.5 & 8.3 & 250.0 & 14.1 & 8.68 & 2.56 & 0.29 & 0.48 & 0.09 & 0.03 & 6.70 & 0.77 & 0.00 & 0.00 \\
\hline D_6 & 8.4 & 4.6 & 6.7 & 0.5 & 298.3 & 3.6 & 260.0 & 14.1 & 8.48 & 2.44 & 0.10 & 0.10 & 0.05 & 0.02 & 6.30 & 1.68 & 0.00 & 0.00 \\
\hline D_7 & 9.0 & 5.0 & 6.3 & 0.2 & 303.5 & 9.9 & 260.0 & 14.1 & 7.95 & 2.88 & 0.18 & 0.23 & 0.09 & 0.03 & 6.38 & 1.88 & 0.00 & 0.00 \\
\hline D_8 & 9.2 & 4.7 & 6.6 & 0.6 & 320.8 & 12.5 & 280.0 & 28.3 & 7.83 & 2.93 & 0.10 & 0.04 & 0.07 & 0.01 & 6.78 & 0.38 & 0.11 & 0.01 \\
\hline E_1 & 7.0 & 5.3 & 6.8 & 0.4 & 278.3 & 7.9 & 225.0 & 19.1 & 8.75 & 2.32 & 0.45 & 0.80 & 0.06 & 0.05 & 5.03 & 1.65 & 0.00 & 0.00 \\
\hline E_2 & 7.4 & 4.9 & 6.6 & 0.4 & 299.8 & 4.6 & 220.0 & 16.3 & 8.88 & 2.26 & 0.39 & 0.68 & 0.07 & 0.05 & 6.30 & 0.47 & 0.00 & 0.00 \\
\hline F_1 & 7.0 & 3.8 & 6.6 & 0.4 & 281.5 & 16.1 & 300.0 & 35.6 & 8.93 & 2.05 & 0.23 & 0.05 & 0.05 & 0.02 & 4.88 & 0.47 & 0.00 & 0.00 \\
\hline
\end{tabular}

Explanations: $\mathrm{T}$ - water temperature $\left({ }^{\circ} \mathrm{C}\right)$; Cond - conductivity $\left(\mathrm{mS} \mathrm{m}^{-1}\right)$; Hard - total hardness $\left(\mathrm{CaCO}_{3} \mathrm{mg} \mathrm{dm}^{-3}\right)$; Oxy - dissolved oxygen $\left(\mathrm{O}_{2} \mathrm{mg} \mathrm{dm}{ }^{-3}\right) ; \mathrm{NH}_{4}-\mathrm{N}-\operatorname{ammonium}\left(\mathrm{NNH}_{4} \mathrm{mg} \mathrm{dm}{ }^{-3}\right) ; \mathrm{NO}_{2}-\mathrm{N}-\operatorname{nitrite}\left(\mathrm{NNO}_{2} \mathrm{mg} \mathrm{dm}^{-3}\right) ; \mathrm{NO}_{3}-\mathrm{N}-$ nitrate $\left(\mathrm{NNO}_{3} \mathrm{mg} \mathrm{dm}^{-3}\right)$; Phos - phosphate $\left(\mathrm{PPO}_{4} \mathrm{mg} \mathrm{dm}{ }^{-3}\right)$.

Table 3. Average abundance of mayfly species in the streams studied in the Pieniny Mts.

\begin{tabular}{|c|c|c|c|c|c|c|c|c|c|c|c|c|c|}
\hline \multirow{2}{*}{ Species } & \multirow[b]{2}{*}{ Abrev. } & \multicolumn{2}{|c|}{ A } & \multicolumn{2}{|c|}{ B } & \multicolumn{2}{|c|}{$\mathrm{C}$} & \multicolumn{2}{|c|}{$\mathrm{D}$} & \multicolumn{2}{|c|}{$\mathrm{E}$} & \multicolumn{2}{|l|}{$\mathrm{F}$} \\
\hline & & Mean & $\mathrm{SD}$ & Mean & $\mathrm{SD}$ & Mean & $\mathrm{SD}$ & Mean & $\mathrm{SD}$ & Mean & $\mathrm{SD}$ & Mean & $\mathrm{SD}$ \\
\hline Baetis alpinus (Pictet, 1843) & Bae_alp & 243.6 & 325.5 & 90.9 & 63.1 & 116.9 & 101.9 & 1050.8 & 1654.6 & 155.0 & 159.8 & 19.0 & - \\
\hline Baetis melanonyx (Pictet, 1843) & Bae_mel & - & - & 4.6 & 12.1 & 14.0 & 34.9 & 9.4 & 18.4 & - & - & - & - \\
\hline Baetis rhodani (Pictet, 1843) & $B a \_r h o$ & 187.6 & 194.2 & 110.7 & 55.4 & 339.0 & 231.3 & 1336.0 & 2700.7 & 38.5 & 26.2 & 65.0 & - \\
\hline Baetis vernus (Curtis, 1834 & Bae_ver & 15.0 & 24.5 & 2.3 & 3.0 & 7.3 & 9.0 & 0.1 & 0.4 & 3.0 & 4.2 & 2.0 & - \\
\hline Baetis muticus (L., 1758) & Ala_mut & 216.0 & 314.0 & 61.6 & 58.9 & 46.6 & 17.5 & 115.6 & 107.4 & 151.5 & 183.1 & - & - \\
\hline Baetis alpinus gr. sp. juv. & $B a e_{-} s p_{\mathbf{\_}} a$ & 6.6 & 14.8 & - & - & - & - & 0.4 & 1.1 & 22.5 & 31.8 & - & - \\
\hline Baetis rhodani gr. sp. juv. & Baet_sp & 2.6 & 5.8 & - & - & - & - & - & - & - & - & 4.0 & - \\
\hline Centroptilum luteolum (O.F. Müller, 1776) & Centr_lu & 0.4 & 0.9 & - & - & - & - & 0.5 & 1.1 & - & - & - & - \\
\hline Epeorus assimilis Eaton, 1885 & Epeo_ass & 0.8 & 1.1 & 0.9 & 1.9 & 1.1 & 1.7 & 2.1 & 3.5 & - & - & - & - \\
\hline $\begin{array}{l}\text { Rhithrogena carpatoalpina } \\
\text { Kłonowska et al., } 1897\end{array}$ & Rhith_ca & - & - & - & - & - & - & 11.0 & 16.0 & - & - & - & - \\
\hline Rhithrogena iridina (Kolenati, 1859) & Rhith_ir & 66.8 & 64.3 & 52.7 & 44.6 & 138.6 & 168.9 & 232.4 & 258.8 & 94.5 & 72.8 & 23.0 & - \\
\hline Rhithrogena semicolorata gr. sp juv. & Rhith_sp & 0.6 & 0.9 & - & - & - & - & 10.0 & 15.4 & - & - & - & - \\
\hline Ecdyonurus subalpinus (Klapálek, 1907) & $E c_{-} s u b$ & 92.2 & 18.6 & 51.1 & 50.5 & 250.6 & 269.6 & 49.0 & 24.5 & 69.0 & 48.1 & 62.0 & - \\
\hline Ecdyonurus submontanus Landa, 1969 & $E c_{-} s m o$ & - & - & - & - & - & - & 1.6 & 2.9 & - & - & - & - \\
\hline Ecdyonurus carpathicus Sowa, 1973 & $E c_{-} c a r$ & - & - & 0.1 & 0.4 & 8.3 & 14.7 & 0.1 & 0.4 & - & - & 1.0 & - \\
\hline Ecdyonurus helveticus gr. sp juv. & $E c_{-} s p_{-} v$ & - & - & - & - & - & - & 0.6 & 1.8 & - & - & - & - \\
\hline Ecdyonurus venosus gr. sp. juv. & $E c_{-} s p_{-} h$ & - & - & - & - & - & - & 1.1 & 2.1 & - & - & - & - \\
\hline Electrogena lateralis (Curtis, 1834) & Ele_ate & - & - & - & - & - & - & 0.3 & 0.7 & - & - & - & - \\
\hline Habrophlebia lauta Eaton, 1884 & $H a b r_{-} l a$ & 51.2 & 104.2 & - & - & 1.0 & 1.4 & 0.6 & 1.8 & - & - & - & - \\
\hline Habroleptoides confusa Sartori et Jacob, 1986 & $H a b r \_c o n$ & 0.6 & 1.3 & 1.1 & 1.5 & 8.1 & 8.7 & 6.1 & 7.7 & 9.0 & 12.7 & - & - \\
\hline Ephemera danica O.F. Müller, 1764 & Ephe_dan & - & - & 0.1 & 0.4 & 2.4 & 3.8 & 1.8 & 4.2 & - & - & - & - \\
\hline Serratella ignita (Poda, 1761) & Serr_ign & - & - & - & - & 13.6 & 32.9 & - & - & - & - & - & - \\
\hline Ephemerella mucronata (Bengtsson, 1909) & Eph_muc & 0.4 & 0.5 & - & - & 0.1 & 0.4 & 11.6 & 20.1 & - & - & - & - \\
\hline Caenis beskidensis Sowa, 1973 & Caen_bes & - & - & - & - & - & - & 1.5 & 2.8 & - & - & - & - \\
\hline
\end{tabular}


Table 4. Ranking of environmental factors of forward selection of canonical correspondence analysis (CCA) for mayfly assemblages for all study sites (undisturbed and disturbed) and sites without human activity (undisturbed). Abbreviations see Table 1.

\begin{tabular}{|c|c|c|c|c|}
\hline Step & Factors & Lambda & Monte Carlo permutation test & Variance explained \\
\hline \multicolumn{5}{|c|}{ Undisturbed and disturbed } \\
\hline 1 & $\mathrm{R}$ & 0.22 & $P=0.002, \mathrm{~F}=7.61$ & 0.22 \\
\hline 2 & $\mathrm{O}$ & 0.12 & $P=0.004, \mathrm{~F}=4.73$ & 0.35 \\
\hline 3 & $\mathrm{~S}$ & 0.08 & $P=0.012, \mathrm{~F}=3.14$ & 0.42 \\
\hline 4 & PG & 0.07 & $P=0.008, \mathrm{~F}=3.19$ & 0.5 \\
\hline 5 & Temp & 0.05 & $P=0.022, \mathrm{~F}=2.44$ & 0.55 \\
\hline \multicolumn{5}{|c|}{ Undisturbed } \\
\hline 1 & PG & 0.2 & $P=0.002, \mathrm{~F}=6.53$ & 0.2 \\
\hline 2 & $\mathrm{C}$ & 0.1 & $P=0.008, \mathrm{~F}=3.56$ & 0.3 \\
\hline 3 & $\mathrm{pH}$ & 0.07 & $P=0.002, \mathrm{~F}=2.79$ & 0.37 \\
\hline 4 & $\mathrm{~S}$ & 0.007 & $P=0.01, \mathrm{~F}=2.93$ & 0.44 \\
\hline 4 & Temp & 0.05 & $P=0.02, \mathrm{~F}=2.32$ & 0.5 \\
\hline 5 & Vel & 0.05 & $P=0.014, \mathrm{~F}=2.32$ & 0.55 \\
\hline 6 & Dis & 0.05 & $P=0.03, \mathrm{~F}=2.26$ & 0.59 \\
\hline 7 & Cond & 0.04 & $P=0.052, \mathrm{~F}=1.96$ & 0.63 \\
\hline
\end{tabular}

ing Principal Component Analysis (PCA) with no transformation of data or sample weights and centering by species.

To determine the relative importance of environmental factors explaining the variation in species density for season and whole data set, forward selection of canonical correspondence analysis (CCA) was used (ter Braak \& Prentice 1988). The statistical significance of each variable selected was judged using a Monte-Carlo permutation test. The ordination analyses were performed with Canoco for Windows v. 4.21 (ter Braak \& Smilauer 2003).

\section{Results}

\section{Species composition}

Nineteen mayfly species were noted in the Pieniny Mts streams studied. The greatest number of mayfly species was noted in the Biały stream (18 species), and the least in the Lonny and Ociemny streams (7 and 6 species). Streams in the Sobczyński and Pieniński catchments had 11 mayfly species each, and the Macelowy stream had 14 species. The main dominants in the streams were Baetis alpinus, Baetis rhodani, Baetis muticus, Rhithrogena iridina and Ecdyonurus subalpinus (Table 3). These include mountain elements (B. alpinus, $R$. iridina) and species that are more widespread ( $B$. rhodani, B. muticus), occurring in the Carpathian Mts at up to $900-1200 \mathrm{~m}$ a.s.l. Some species were noted at a limited number of sites, and in low numbers, e.g., Centroptilum luteolum, Ecdyonurus submontanus, Electrogena lateralis, Serratella ignita and Caenis beskidensis. E. submontanus and C. beskidensis, occurring at $250-$ $800 \mathrm{~m}$. a.s.l., are associated with small rivers at lower altitudes. C. luteolum, E. lateralis, and S. ignita are present in various types of running waters, at a wide range of elevations.

A non-metric multidimensional scaling performed on the Bray-Curtis matrix of similarity of 30 assemblages indicated high fit of assemblages on the first two dimensions (final stress $=0.10$ ). The two first axes of the PCA explained 100 per cent of the total variance of similarity matrix. The first axis accounted for $78.6 \%$ of

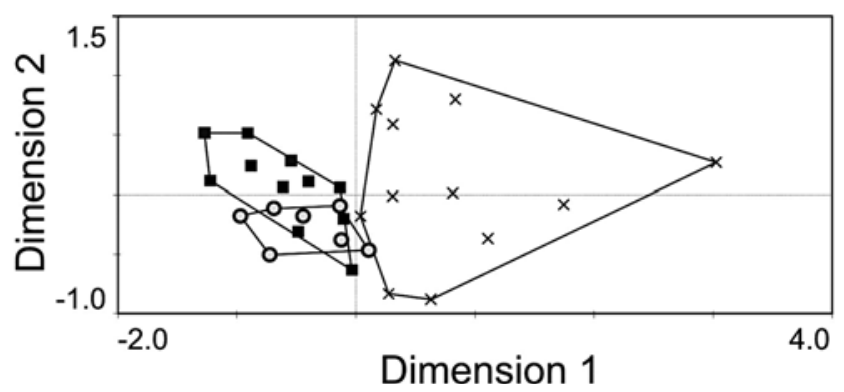

Fig. 2. Non-metric multidimensional scaling showing mayfly species composition. Assemblages were classified into groups located in lower (X), middle (circles) and upper (squares) parts of the streams. The final stress of the NMDS solution was 0.10 .

the total variance clearly divide assemblages from lower - anthropogenic and upper more natural parts of the streams (Fig. 2).

Mayfly communities distribution and abiotic parameters

The main factors accounting for the description of the mayfly communities in the Pieniny region were those connected with human activity: stream regulation $(\mathrm{R})$ and organic pollution $(\mathrm{O})$ (Table 4). Other significant factors are substrate type - pebble and gravel (PG), riparian vegetation - shrubs $(\mathrm{S})$; and physiochemical parameters $-\mathrm{pH}$ and water temperature $(\mathrm{T})$. The first two CCA axes described $41.8 \%$ variance of species data and $75.5 \%$ variance of species-environment relations (Fig. 3A). Substrate type correlated positively $(\mathrm{R}=$ $0.81)$ while stream regulation $(\mathrm{R})$ and organic pollution $(\mathrm{O})$ correlated negatively $(\mathrm{R} 1=-0.87, \mathrm{R} 2=-0.58$, respectively) with the first axis. Most of the mayfly species were concentrated on pebble and gravel sites, but E. lateralis and B. rhodani preferred more anthropogenic sites (Fig. 3A). When we considered only undisturbed sites, the most significant factors were $\mathrm{pH}$; substrate type - cobble $(\mathrm{C})$, pebble and gravel $(\mathrm{PG})$; distance from the source (Dis); current velocity (Vel); and riparian vegetation - shrubs (S) (Table 4). Also sig- 

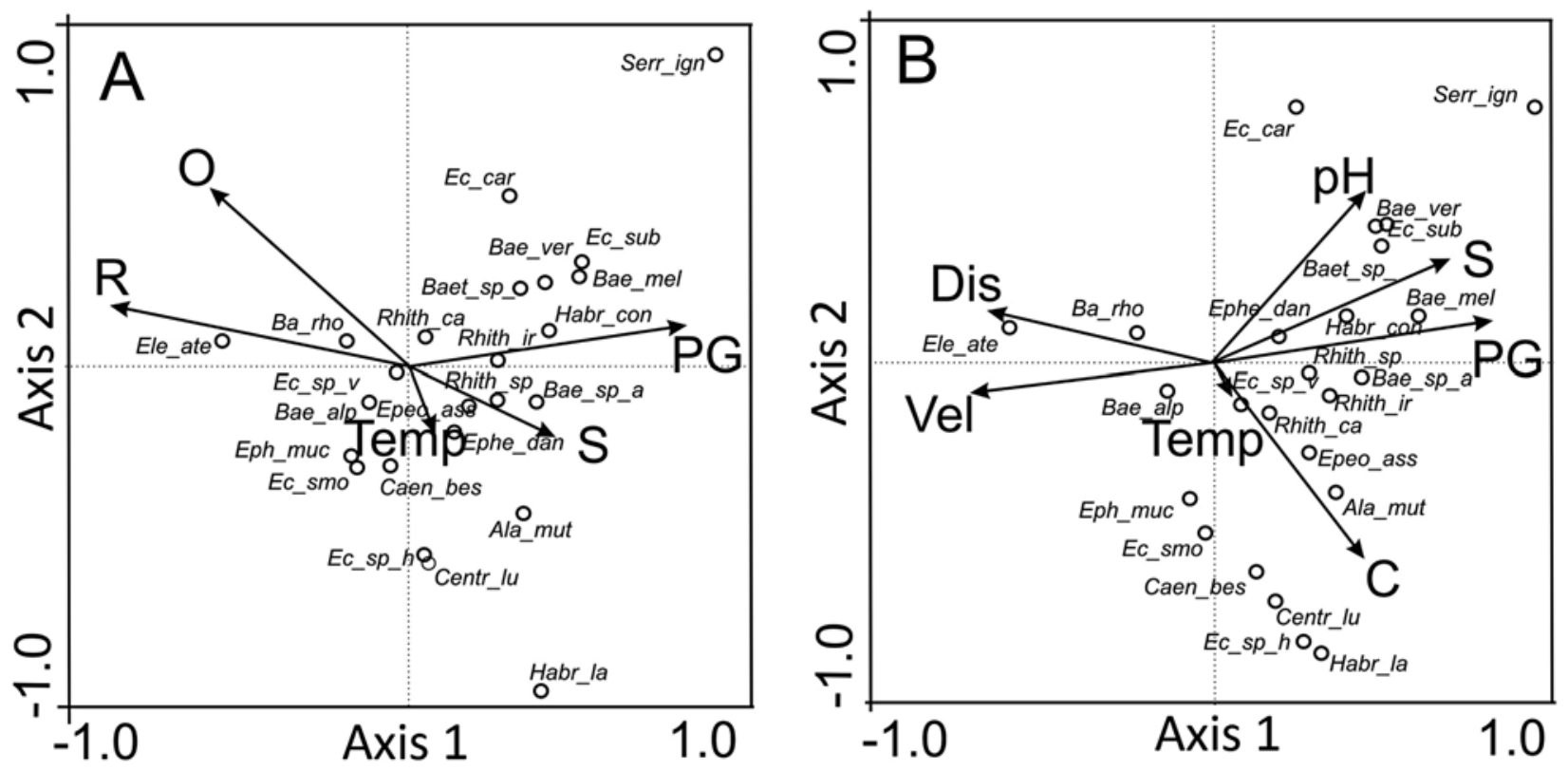

Fig. 3. Diagram of forward selection of canonical correspondence analysis (CCA) for mayfly communities in environmental gradients: A - all study sites included; B - only undisturbed sites included (for description of variables see Table 1).

Table 5. Ranking of environmental factors derived from forward selection of canonical correspondence analysis (CCA) for seasons of mayfly communities. Abbreviations see Table 1.

\begin{tabular}{|c|c|c|c|c|}
\hline Step & Factors & Lambda & Monte Carlo permutation test & Variance explained \\
\hline \multicolumn{5}{|c|}{ Early spring } \\
\hline 1 & Phos & 0.12 & $P=0.02, \quad \mathrm{~F}=3.34$ & 0.12 \\
\hline 2 & Dis & 0.1 & $P=0.012, \mathrm{~F}=2.87$ & 0.22 \\
\hline 3 & $\mathrm{pH}$ & 0.1 & $P=0.008, \mathrm{~F}=3.26$ & 0.32 \\
\hline 4 & $\mathrm{~S}$ & 0.11 & $P=0.002, \mathrm{~F}=3.72$ & 0.43 \\
\hline 5 & $\mathrm{Vel}$ & 0.09 & $P=0.014, \mathrm{~F}=3.35$ & 0.52 \\
\hline 6 & $\mathrm{PG}$ & 0.07 & $P=0.016, \mathrm{~F}=2.65$ & 0.58 \\
\hline 7 & $\mathrm{C}$ & 0.09 & $P=0.002, \mathrm{~F}=3.95$ & 0.67 \\
\hline 8 & $\mathrm{Ni}$ & 0.06 & $P=0.024, \mathrm{~F}=2.85$ & 0.73 \\
\hline 9 & $\mathrm{~F}$ & 0.04 & $P=0.076, \mathrm{~F}=2.01$ & 0.77 \\
\hline \multicolumn{5}{|c|}{ Late spring } \\
\hline 1 & $\mathrm{R}$ & 0.29 & $P=0.002, \mathrm{~F}=7.18$ & 0.29 \\
\hline 2 & $\mathrm{~S}$ & 0.12 & $P=0.04, \quad \mathrm{~F}=3.25$ & 0.41 \\
\hline 3 & Temp & 0.06 & $P=0.15, \quad F=1.52$ & 0.47 \\
\hline \multicolumn{5}{|c|}{ Summer } \\
\hline 1 & $\mathrm{~S}$ & 0.15 & $P=0.042, \mathrm{~F}=2.33$ & 0.15 \\
\hline 2 & $\mathrm{~F}$ & 0.14 & $P=0.018, \mathrm{~F}=2.39$ & 0.29 \\
\hline 3 & Dep & 0.13 & $P=0.09, \quad \mathrm{~F}=2.35$ & 0.42 \\
\hline 4 & Vel & 0.1 & $P=0.116, \mathrm{~F}=1.94$ & 0.52 \\
\hline \multicolumn{5}{|c|}{ Autumn } \\
\hline 1 & $\mathrm{C}$ & 0.29 & $P=0.002, \mathrm{~F}=4.62$ & 0.29 \\
\hline 2 & $\mathrm{R}$ & 0.21 & $P=0.004, \mathrm{~F}=3.61$ & 0.5 \\
\hline 3 & G & 0.15 & $P=0.022, \mathrm{~F}=2.70$ & 0.64 \\
\hline 4 & Alt & 0.16 & $P=0.002, \mathrm{~F}=3.25$ & 0.81 \\
\hline 5 & $\mathrm{~B}$ & 0.12 & $P=0.044, \mathrm{~F}=2.58$ & 0.93 \\
\hline 6 & Cond & 0.11 & $P=0.026, \mathrm{~F}=2.52$ & 1.04 \\
\hline
\end{tabular}

nificant were water temperature $(\mathrm{T})$ and conductivity (Cond). The first two ordination axes described $42.1 \%$ variance of species data and $74.4 \%$ of the variance between species and environment. There were three groups of factors responsible for mayfly distribution (Fig. 3B). The first group - presence of pebble and gravel, presence of shrubs, and increasing $\mathrm{pH}$ - corre- lated significantly with the first canonical axis $(\mathrm{R} 1=$ $0.81, \mathrm{R} 2=0.69, \mathrm{R} 3=0.45)$, describing most of the mayfly species variation (Fig. 3B). Higher discharge and increasing velocity correlating negatively with the first axis $(\mathrm{R} 1=0.71, \mathrm{R} 2=0.66)$ eliminated most of the species and provided better conditions for species specific to disturbed areas. The third group of environ- 

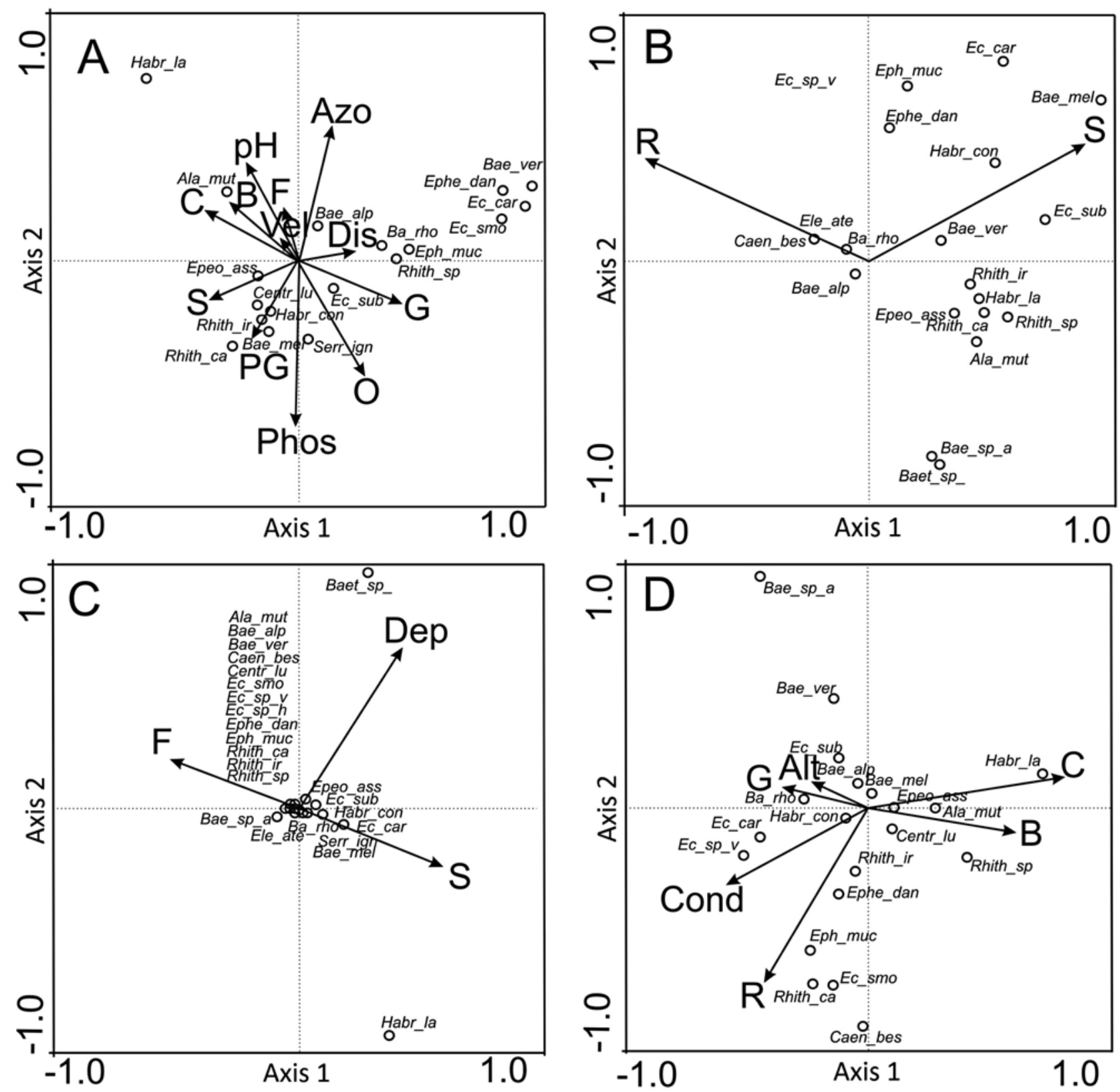

Fig. 4. Variation of species composition in relation to environmental factors for mayfly communities: A - early spring; B - late spring; C - summer; D - autumn (for description of variables see Table 1).

mental factors, $\%$ of cobbles and temperature, correlated negatively with the second ordination axis (R1 $=-0.57, \mathrm{R} 2=-0.5)$ and creates favourable conditions for such species as C. beskidensis or Habrophlebia lauta (Fig. 3B).

Seasonal response of mayfly communities to environmental factors

CCA analysis of mayfly communities and environmental characteristics in different seasons of the year revealed high variation in occurrence of mayfly species depending on the season (Table 5). Only in early spring and autumn did mayfly communities occur that were dependent on many environmental factors. The most significant environmental factors for early spring mayfly communities were phosphate (Phos); substrate type - cobble $(\mathrm{C})$ and pebble and gravel $(\mathrm{PG}) ; \mathrm{pH}$; distance from source (Dis); nitrite $\left(\mathrm{NO}_{2}-\mathrm{N}\right)$; and riparian vegetation - shrubs (S) and forest (F) (Table 5). The first two axes describing $40 \%$ of species variance ordinated mayfly species into three main groups (Fig. 4A). The first depended on higher discharge, the second relied on substrate and presence of shrubs, and the third correlated with $\mathrm{pH}$ and \% of boulders and cobbles. During late spring and summer only a few factors described the variation in the mayfly communities: stream regulation $(\mathrm{R})$ and shrubs $(\mathrm{S})$ in late spring, and riparian vegetation - shrubs (S), forest $(\mathrm{F})$ - and water depth (Dep) in summer (Table 5, Figs 4B, C). Stabilization and differentiation of mayfly communities which were dependent on many environmental factors took place in autumn. The most significant factors for autumn mayfly com- 
munities were substrate type - cobble $(\mathrm{C})$, pebble and gravel (PG) and boulder (B); altitude (Alt); stream regulation $(\mathrm{R})$; riparian vegetation - grasses $(\mathrm{G})$; and water conductivity (Cond) (Table 5, Fig. 4D). The main factors responsible for describing most of the variation were large amounts of cobbles (C) and boulders (B) (weighted correlations with the first axis are $\mathrm{R} 1=0.81$, $\mathrm{R} 2=0.6$, respectively) .

\section{Habitat preferences of mayflies}

In the mayfly communities it is possible to distinguish species occurring widely, at many sites, as well as species associated only with certain localities (Table 3 ). $B$. alpinus was present at all 30 sites in the streams studied. B. rhodani and E. subalpinus were present at 28 sites. Two more species, A. muticus and R. iridina, were observed at fewer sites. A contrasting group was composed of species occurring at only a few sites (1-3). These include C. luteolum, E. submontanus, E. lateralis and $C$. beskidensis, which were noted at only a few sites in the lower course of the Bialy stream. S. ignita was observed only in the Macelowy stream, at sites in the lower course. A separate group was composed of species present in a few communities in the study area. Examples of these are Rhithrogena carpatoalpina, Ecdyonurus carpathicus, Habrophlebia lauta, Ephemera danica and Ephemerella mucronata.

\section{Discussion}

Mayfly communities and environmental parameters Macroinvertebrate diversity has been shown to increase with stream size (Breitenmoser-Würsten \& Sartori 1995; Heino et al. 2005; Paller et al. 2006). The number of mayfly species noted in the streams of the Pieniny Mts is characteristic of headwater mountain streams in the rhithral zone. A maximum of 15-20 mayfly species can be expected in this type of stream (Bauernfeind \& Moog 2000). According to Sowa (1975) and Svitok (2006) the streams of the Pieniny Mts are located within two zones (Fig. 2). Stream zone no 1 includes the initial reach of the stream (eucrenal-hypocrenal), whose sources are about $700 \mathrm{~m}$ a.s.l. Stream zone 2 begins $0.7-1.5 \mathrm{~km}$ from the sources, at $500-800 \mathrm{~m}$ a.s.l., and ends $2.5-9 \mathrm{~km}$ from the sources.

Mayfly communities of the lower parts of the streams differ from those of the other catchment areas studied because of human activity, particularly stream regulation and organic pollution (Fig. 2, Table 4). Mayflies are a highly sensitive group and react to any changes in the ecosystem, which is why they are often used in biomonitoring of running waters (Soldán et al. 1998). Only two species, E. lateralis and B. rhodani, show positive correlation with stream regulation and organic pollution (Fig. 3A). Regulated reaches seem to be the best mesohabitat for E. lateralis, while $B$. rhodani is able to adapt to variable environmental conditions and can occur along virtually the entire course of the stream (Sowa 1975; Soldán et al. 1998).
A significant factor for mayfly communities composition and relative abundance in the Pieniny Mountain sites and in the undisturbed sites was values of $\mathrm{pH}$. This water parameter has been mentioned as one of the most important for macroinvertebrate communities (Zamora-Muńoz et al. 1993; Svitok 2006), although some authors believe that only extreme $\mathrm{pH}$ values significantly affect Ephemeroptera (Soldán et al. 1998). Substrate type was a significant parameter for mayfly communities both at all sites, undisturbed and disturbed. Substrate type is known to be one of the main factors influencing macroinvertebrate richness and community structure (Ward 1992; Townsend et al. 2003; Allan \& Castillo 2007). In the streams of the Pieniny Mts the proportion of gravel and cobble are most important for mayfly communities as it has been noted by the other studies (Füreder et al. 2002; Hieber et al. 2005; Effenberger et al. 2006). Suitable substrate size determines the occurrence of particular mayfly species (Brittain 1982). Cobble, pebble and gravel exhibit greater diversity of macroinvertebrate taxa and species than substrates with smaller particle size (Ward 1992). Distance from source (Dis) and current velocity (Vel) were found to be significant factors for mayfly communities at undisturbed sites during the entire study period. Distance from source indirectly determines stream size and correlates with macroinvertebrate taxa richness (Lorenz et al. 2004). In most mountain streams, macroinvertebrate diversity increases with stream size (Heino et al. 2005; Krno et al. 2007). In this study stream size has also been shown to correlate positively with the number of Ephemeroptera, Plecoptera and Trichoptera species, in both undisturbed and disturbed localities (Paller et al. 2006). In the catchment areas studied in the Pieniny Mts, riparian vegetation was found to be significant for mayfly communities as well as in earlier investigations from the other regions (Townsend et al. 2003). The presence of shrubs (S) and forest (F) on stream banks was significant for all sites, both undisturbed and disturbed, for the entire study period as well as for particular seasons. Riparian vegetation is vital for maintaining channel morphology and ensuring suitable water temperature and amount of light; it also forms a buffer area for the stream (Allan \& Castillo 2007). Moreover, it supplies nutrients and organic matter (litter and wood) to the stream ecosystem (Balestrini et al. 2004), and thus has an important role in determining the trophic structure in the stream. Particular emphasis has been placed on the role of riparian vegetation in mountain streams in naturally forested catchments, where it is often the most important factor differentiating macroinvertebrate occurrence in the stream (Vannote et al. 1980; Füreder et al. 2002; Krno et al. 2007).

The mayfly communities in the streams of the Pieniny Mts varied substantially in different seasons. Only in early spring and autumn were communities observed that were stable and dependent on many different environmental factors. Hieber et al. (2005) point out the considerable fluctuations in environmental factors, especially discharge, and the high degree of tur- 
bulence in the water flow in mountain streams. Discharge of high flow can undoubtedly be a factor contributing to natural disturbances for aquatic insects, and is negatively associated with taxa richness (Ward 1992; Effenberger et al. 2006). It seems that the low degree of order in mayfly communities in late spring and summer is connected with frequent flooding and increased discharge resulting from melting snow, as well as from the high flows that occur frequently in summer in the Carpathians due to heavy rainfall. Such catastrophic flooding occurs in the Pieniny Mts from March to August, and the geological structure of the land, together with the geomorphology of the catchment area and streams, leads to high discharge (Kostarkiewicz 1982). Krno et al. (2006, 2007) observed an increase in discharge in Carpathian mountain streams in spring due to melting of the snow cover. This resulted in higher levels of phosphorus, leading to increased periphyton production. Thus the increase in phosphorus was the effect of natural process of eutrophication, not organic pollution. Higher levels of ammonium originating in the catchment were also observed. This is the effect of more intensive decomposition of organic matter rich in nitrogen compounds, which is washed out of the catchment area when the snow melts in spring. Krno (2007) demonstrates a connection between stabilization of stream conditions in autumn and stabilization of discharge. Kownacki et al. (1997) report an increase in phosphorus and nitrate concentrations in a small mountain stream in the Tatra Mts. The influence of phosphorus and nitrate on mayfly communities is also observed in the streams of the Pieniny Mts in early spring, while in autumn stabilization of communities follows stabilization of the bottom substrate, which results from stabilization of discharge.

\section{Conclusions}

Headwater streams of the Pieniny Mts have a distinctive habitats with well-preserved mayfly communities. Determination of stream characteristics in connection with mayfly communities makes is possible to estimate reference conditions for the type of stream found in the Pieniny Mts, which is important for biological conservation and biomonitoring.

\section{References}

Allan J. D. \& Castillo M.M. 2007. Stream Ecology. Structure and Function of Running Waters. $2^{\text {nd }}$ ed. Springer, Dordrecht, Netherlands, 436 pp. ISBN: 978-1-4020-5583-6

Balestrini R., Cazzola M. \& Buffagni A. 2004. Characterizing hydromorphological features of selected Italian rivers: a comparative application of environmental indices. Hydrobiologia $\mathbf{5 1 6}$ (1-3): 365-379. DOI: 10.1023/B:HYDR.0000025276.19872.ee

Bauernfeind E. \& Moog O. 2000. Mayflies (Insecta: Ephemeroptera) and the assessment of ecological integrity: a methodological approach. Hydrobiologia 422/423 (0): 71-83. DOI: 10.1023/A:1017090504518

Breitenmoser-Würsten C. \& Sartori M. 1995. Distribution, diversity, life cycle and growth of a mayfly community in a prealpine stream system (Insecta, Ephemeroptera). Hydrobiologia 308 (2): 85-101. DOI: 10.1007/BF00007393

Brittain J.E. 1982. Biology of mayflies. Ann. Rev. Entomol. 27: 119-147. DOI: 10.1146/annurev.en.27.010182.001003

Bunn S.E. \& Arthington A.H. 2002. Basic principles and ecological consequences of altered flow regimes for aquatic biodiversity. Environ. Manage. 30 (4): 492-507. DOI: 10.1007/s00267-002-2737-0

Burian S. 1997. An analysis of the distribution and diversity of the Ephemeroptera of Mine, USA, pp. 127-138. In: Landolt P. \& Sartori M. (eds), Ephemeroptera and Plecoptera. Biology - Ecology - Systematics, MTL, Fribourg, Switzerland, 569 pp. ISBN: 2940187010, 9782940187010

Death R.G. \& Joy M.K. 2004. Invertebrate community structure in streams of the Manawatu-Wanganui region, New Zealand: the roles of catchment versus reach scale influences. Freshwater Biol. 49 (8): 982-997. DOI: 10.1111/j.13652427.2004.01243.x

Effenberger M., Sailer G., Townsed C.R. \& Matthaei C.D. 2006. Local disturbance history and habitat parameters influence the microdistribution of stream invertebrates. Freshwater Biol. 51 (2): 312-332. DOI: 10.1111/j.1365-2427.2005.01502.x

Finn D.S. \& Poff N.L. 2005. Variability and convergence in benthic communities along the longitudinal gradients of four $\overline{\text { physically similar }}$ Rocky Mountain streams. Freshwater $\overline{\text { Biol. }}$ 50 (2): $243-261$. DOI: $10.1111 / \mathrm{j} .1365-2427.2004 .01320 . x$

Füreder L., Vacha C., Amprosi K., Bühler S., Hansen C.M.E. \& Mortiz C. 2002. Reference conditions of Alpine streams. Physical habitat and ecology. Water Air Soil Pollut. 2 (2): 279-294. DOI: 10.1023/A:1020171129760

Gordon N.D., McMahon T.A. \& Finlayson B.L. 1994. Stream Hydrology, An Introduction for Ecologist. Wiley \& Sons, New York, 526 pp. ISBN: 0471955051, 9780471955054

Hawkins C.P., Murphy M.L. \& Anderson N.H. 1982. Effects of canopy, substrate composition and gradient on the structure of macroinvertebrate communities in Cascade Range streams of Oregon. Ecology 63 (6): 1840-1856. DOI: 10.2307/1940125

Heino J., Parviainen J., Paavola R., Jehle M., Louhi P. \& Muotka T. 2005. Characterising macroinvertebrate assemblage structure in relation to stream size and tributary position. Hydrobiologia 539 (1): 121-130. DOI: 10.1007/s10750-004-3914-3

Hieber M., Robinson T., Uehlinger U. \& Ward J.V. 2005. A comparison of benthic invertebrate assemblages among different types of alpine streams. Freshwater Biol. 50 (12): 2087-2100. DOI: $\frac{10}{10} \cdot 1111 / \mathrm{j} .1365-2427.2005 .01460 . \mathrm{x}$

Johnson L. \& Gage S. 1997. Landscape approaches to the analysis of aquatic ecosystem. Freshwater Biol. 37 (1): 113-132. DOI: 10.1046/j.1365-2427.1997.00156.x

Kłonowska-Olejnik M. 2000. Jetki (Ephemeroptera) [Mayflies (Ephemeroptera)], pp. 137-141. In: Razowski J. (ed.), Flora i fauna Pienin. Monografie Pienińskie. Tom 1. [Flora and fauna of the Pieniny Mountains] Pieniński Park Narodowy, Krościenko nad Dunajcem, 333 pp.

Kostarkiewicz L. 1982. Hydrography, pp. 70-93. In: Zarzycki K. (ed.), Pieniny. Przyroda $\mathrm{w}$ obliczu zmian [The Nature of the Pieniny Mountains in the face of changes], PAN, Zakład Ochrony Przyrody i Zasobów Naturalnych, PWN, WarszawaKraków, 578 pp. ISBN: 83010325029788301032500

Kownacki A., Dumnicka E., Galas J., Kawecka B. \& Wojtan K. 1997. Ecological characteristics of a high mountain lake outlet stream (Tatra Mts, Poland). Arch. Hydrobiol. 139 (1): 113128.

Krno I., Šporka F., Sttefková E., Tirjaková E., Bitušik P., Bulánková E., Lukáš J., Illešová D., Derka T., Tomajka J. \& Černý J. 2006. Ecological study of a high-mountain stream ecosystem (Hincov potok, High Tatra Mountains, Slovakia). Acta Soc. Zool. Bohem. 69: 299-316.

Krno I., Šporka F., Pastuchová Z., Derka T., ČiamporováZatovičová Z., Bulánková E., Hamerlík L. \& Illešová D. 2007. Assessment of the ecological status of streams in two Carpathian subregions. Int. Rev. Hydrobiol. 92 (4-5): 564581. DOI: $10.1002 /$ iroh. 200610996

Lenzi M.A. \& Comiti F. 2003. Local scouring and morphological adjustments in steep channels with check-dam sequences. 
Geomorphology 55 (1-4): 97-109. DOI: 10.1016/S0169555X(03)00134-X

Lorenz A., Feld C.K. \& Hering D. 2004. Typology of streams in Germany based on benthic invertebrates. Ecoregions, zonation, geology and substrate. Limnologica 34 (4): 379-389. DOI: $10.1016 /$ S0075-9511(04)80007-0

Minshall G.W., Petersen R.C. \& Nimz C.F. 1985. Species richness in streams of different size from the same drainage basin. Am. Nat. 125 (1): 16-38. DOI: 10.1086/284326

Paller M.H., Specht W.L. \& Dyer S.A. 2006. Effects of stream size on taxa richness and other commonly used benthic bioassessment metrics. Hydrobiologia 568 (1): 309-316. DOI: $10.1007 / \mathrm{s} 10750-006-0208-y$

Roth N.E., Allan J.D. \& Erickson D.L. 1996. Landscape influences on stream biotic integrity assessed at multiple spatial scales. Landscape Ecol. 11 (3): 141-156. DOI: 10.1007/BF02447513

Šmilauer P. 2002. WinKyst 1.0, Ceske Budejovice, Czech Republic. http://www.canodraw.com/winkyst.htm (accessed 15.01.2013)

Soldán T., Zahrádková S., Helešic J., Dušek L. \& Landa V. 1998. Distributional and quantitative patterns of Ephemeroptera and Plecoptera in the Czech Republic: a possibility of detection of long term environmental changes of aquatic biotopes. Folia Fac. Sci. Nat. Univ. Masaryk. Brun. Biol. 98, 298 pp. ISBN: $80-210-1870-4$

Sowa R. 1975. Ecology and biogeography of mayflies (Ephemeroptera) of running waters in the Polish part of the Carpathians. Distribution and quantitative analysis. Acta Hydrobiol. 17 (3): 223-297.
Svitok M. 2006. Structure and spatial variability of mayfly (Ephemeroptera) communities in the upper Hron River basin. Biologia 61 (5): 547-554. DOI: 10.2478/s11756-006-0089-6

ter Braak C.F.J. \& Prentice I.C. 1988. A theory of gradient analysis. Adv. Ecol. Res. 18: 271-317. DOI: 10.1016/S00652504(03)34003-6

ter Braak C.F.J. \& Šmilauer P. 2003. CANOCO Reference manual and user's guide to Canoco for Windows. Software for Canonical Community Ordination (Version 4.52). Microcomputer Power, Ithaca, New York, 353 pp.

Townsend C.R., Dolédec S., Norris R., Peacock K. \& Arbuckle C. 2003. The influence of scale and geography on relationships between stream community composition and landscape variables. description and prediction. Freshwater Biol. 48 (5): 768-785. DOI: 10.1046/j.1365-2427.2003.01043.x

Ward J.V. 1992. Aquatic Insect Ecology. Biology and Habitat. John Wiley \& Sons, New York-Singapore, 438 pp. ISBN: 0471550078, 9780471550075

Vannote R.L., Minshall G.W., Cummins K.W., Sedell J.R. \& Cushing C.E. 1980. The river continuum concept. Can. J. Fish. Aquat. Sci. 37 (1): 130-137. DOI: 10.1139/f80-017

Wolman M.J. 1954. A method of sampling coarse river bed material. Transactions, American Geophysical Union 35 (6): 951-956. DOI: 10.1029/TR035i006p00951

Zamora-Muñoz C., Sanchez-Ortega A. \& Alba-Tercedor J. 1993. Physicochemical factors that determine the distribution of mayflies and stoneflies in a high-mountain stream in Southern Europe (Sierra Nevada, Southern Spain). Aquat. Insects 15 (1): 11-20. DOI: 10.1080/01650429309361495

Received February 14, 2013 Accepted December 20, 2013 